\title{
Joint 3D Trajectory Design and Time Allocation for UAV- Enabled Wireless Power Transfer Networks
}

DOI:

10.1109/TVT.2020.2972133

\section{Document Version}

Accepted author manuscript

Link to publication record in Manchester Research Explorer

\section{Citation for published version (APA):}

Feng, W., Zhao, N., Shaopeng, A., Tang, J., Zhang, X., Fu, Y., So, D. K. C., \& Wong, K-K. (2020). Joint 3D

Trajectory Design and Time Allocation for UAV-Enabled Wireless Power Transfer Networks. IEEE Transactions on Vehicular Technology. https://doi.org/10.1109/TVT.2020.2972133

\section{Published in:}

IEEE Transactions on Vehicular Technology

\section{Citing this paper}

Please note that where the full-text provided on Manchester Research Explorer is the Author Accepted Manuscript or Proof version this may differ from the final Published version. If citing, it is advised that you check and use the publisher's definitive version.

\section{General rights}

Copyright and moral rights for the publications made accessible in the Research Explorer are retained by the authors and/or other copyright owners and it is a condition of accessing publications that users recognise and abide by the legal requirements associated with these rights.

\section{Takedown policy}

If you believe that this document breaches copyright please refer to the University of Manchester's Takedown Procedures [http://man.ac.uk/04Y6Bo] or contact uml.scholarlycommunications@manchester.ac.uk providing relevant details, so we can investigate your claim.

\section{OPEN ACCESS}




\title{
Joint 3D Trajectory Design and Time Allocation for UAV-Enabled Wireless Power Transfer Networks
}

\author{
Wanmei Feng, Nan Zhao, Senior Member, IEEE, Shaopeng Ao, Jie Tang, Senior Member, IEEE, \\ Xiuyin Zhang, Senior Member, IEEE, Yuli Fu, Daniel K. C. So and Kai-Kit Wong, Fellow, IEEE
}

\begin{abstract}
This paper considers a rotary-wing unmanned aerial vehicle (UAV)-enabled wireless power transfer system, where a UAV is dispatched as an energy transmitter (ET), transferring radio frequency (RF) signals to a set of energy receivers (ERs) periodically. We aim to maximize the energy harvested at all ERs by jointly optimizing the UAV's three-dimensional (3D) placement, beam pattern and charging time. However, the considered optimization problem taking into account the drone flight altitude and the wireless coverage performance is formulated as a non-convex problem. To tackle this problem, we propose a low-complexity iterative algorithm to decompose the original problem into four sub-problems in order to optimize the variables sequentially. In particular, we first use the sequential unconstrained convex minimization based algorithm to find the globally optimal UAV two-dimensional (2D) position. Subsequently, we can directly obtain the optimal UAV altitude as the objective function of problem is monotonic decreasing with respect to UAV altitude. Then, we propose the multiobjective evolutionary algorithm based on decomposition (MOEA/D) based algorithm to control the phase of antenna array elements, in order to achieve high steering performance of multi-beams. Finally, with the above solved variables, the original problem is reformulated as a single-variable optimization problem where charging time is the optimization variable, and can be solved using the standard convex optimization techniques. Furthermore, we use the branch and bound method to design the UAV trajectory which can be constructed as traveling salesman problem (TSP) to minimize flight distance. Numerical results validate the theoretical findings and demonstrate that significant performance gain in terms of sum received power of ERs can be achieved by the proposed algorithm in UAV-enabled wireless power transfer networks.
\end{abstract}

Index Terms-Multi-beam, trajectory optimization, UAV 3D placement, wireless power transfer.

This paper will be presented in part at the IEEE 91st Vehicular Technology Conference (VTC 2020-Spring), Antwerp, Belgium, 2020. This work has been supported in part by the National Natural Science Foundation of China under Grant 61971194 and 61871065, in part by the Natural Science Foundation of Guangdong Province under Grant 2017A030313383 and 2019A1515011607, in part by the Open Research Fund of National Mobile Communications Research Laboratory, Southeast University (No. 2019D06), and in part by the Fundamental Research Funds for the Central Universities under Grant 2019JQ08. (Corresponding author: Jie Tang.)

W. Feng, S. Ao, J. Tang, X. Zhang and Y. Fu are with the School of Electronic and Information Engineering, South China University of Technology, Guangzhou, China. (e-mail: eewmfeng@mail.scut.edu.cn; 1472152862@qq.com; eejtang@scut.edu.cn; zhangxiuyin@scut.edu.cn and fuyuli@scut.edu.cn).

N. Zhao is with the School of Information and Communication Engineering, Dalian University of Technology, Dalian, China. (e-mail: zhaonan@dlut.edu.cn).

D. K. C. So is with the School of Electrical and Electronic Engineering, University of Manchester, Manchester, United Kingdom. (e-mail: d.so@manchester.ac.uk).

K.-K Wong is with the Department of Electronic and Electrical Engineering, University College London, London, United Kingdom. (e-mail: kaikit.wong@ucl.ac.uk).

\section{INTRODUCTION}

$\mathbf{T}$ HE Internet of Things (IoT) exploit the interconnection between heterogeneous smart devices to collect and exchange data, which can be used in smart home, intelligent transportation system (ITS) and smart city [1]-[5]. In particular, the third generation $(3 \mathrm{G})$ and the fourth generation (4G) cellular networks are the two communication techniques to support the IoT connectivity and provide wide coverage area, low deployment cost and high security communications [6]. However, both of these techniques can not support effectively Machine-Type communications (MTC) due to many requirements for MTC devices, such as high data rate, lowlatency, low power consumption, high security and network scalability and coverage. The fifth generation (5G) mobile networks are capable of overcoming the limitations of the current technologies, which provides Gigabit data rate and low latency communications to connected devices [7]-[12]. As a result, $5 \mathrm{G}$ enabled IoT has attracted a lot of attention in the research community and industry.

In fact, 5G networks connect massive IoT applications which causes enormous power consumption. Wireless power transfer (WPT) and energy harvesting (EH) have been considered as promising techniques to prolong battery-life in 5G and beyond [13]. Particularly, inductive coupling and magnetic resonance coupling are two types of WPT technologies, but can only transfer power over several centimeters and several meters, respectively, which are not possible yet for longrange wireless charging. On the other hand, radio frequency (RF) energy transfer technique conveys energy contained in electromagnetic waves $(3 \mathrm{kHz} \sim 300 \mathrm{GHz})$ to the electronics devices [14]. Since the distance between transmitters and receivers can be up to several kilometers, RF energy transfer technique is suitable for far-field wireless energy transfer. Thus, RF-enabled WPT has attracted much attention in both academia and industry recently, and adopted in many applications. However, the ETs may be far away from the energy receivers (ERs) when they are in disaster areas or remote mountain areas, and thus the receivers sustain low energy transfer efficiency due to the path loss effect.

Unmanned aerial vehicles (UAVs), owning to their autonomy, flexibility, mobility, can rapidly deploy in rural and geographically constrained areas and provide reliable and cost-effective wireless connectivity instead of the local base stations. Hence, due to many advantages of UAVs, the notion of UAV-enabled wireless power transfer has been proposed in recent years. In practice, the UAV flight duration is finite 
due to the limitation of the onboard battery capacity, and hence, minimizing the aerodynamic power consumption or maximizing the energy efficiency of UAVs have been studied by several existing works [15]-[19]. In [15], the authors maximized the UAV's energy efficiency via designing flight trajectory by jointly considering throughput and propulsion energy consumption. In [16], the authors studied the UAV trajectory to maximize energy transfer subject to the maximum UAV flying speed in UAV-enabled WPT networks. In [17], the authors maximized the minimum uplink throughput by jointly optimizing UAV trajectory and transmission resource allocations for UAV-enabled wireless powered communication networks (WPCN). Based on the aforementioned researches on two-dimensional (2D) location of UAVs, some existing works take into account flight altitude which is related to ground coverage area. In [18], the authors studied 3D placement of a UAV to maximize the number of covered users under the constraint of minimum transmit power. In [19], the authors jointly optimized altitude, beamwidth, location and bandwidth to minimize the sum power of UAV-enabled wireless communications.

The main practical challenge for implementing RF-based WPT is the low energy transfer efficiency caused by power attenuation due to the path loss. One suitable solution is to use energy beamforming techniques. Specifically, energy transmitters with antenna array form an energy beam towards the corresponding energy receivers to maximize the received signal strength, which can improve the energy transfer efficiency due to the increase of effective aperture area ${ }^{1}$. For example, the authors in [21] proposed two practical channel training methods where ETs transmit beamformed energy to a single-antenna ER for receiving energy feedback. In [22], ETs generate energy beamforming to ERs in order to obtain energy measurement feedback by three channel acquisition methods in multiple-input-multiple-output (MIMO) systems.

\section{A. Main contributions}

Previous works in the literature designed the 2D trajectory planning for UAVs that maximized the energy efficiency in UAV-aided wireless communication networks [15], UAVenabled WPT networks [16] and WPCN [17], where UAV altitude is not considered to ensure the coverage areas of UAVs. On the other hand, the works in [18], [19] investigated the 3D placement of UAVs to maximize the wireless coverage, but not consider the trajectory planning for UAVs to minimize the flight distance. In contrast to the previous literature [15][19], in this paper, we investigate an energy harvesting optimization problem for UAV-enabled WPT networks that jointly optimizes UAV's 3D placement, beam pattern, charging time and $3 \mathrm{D}$ trajectory. To satisfy the requirement that the UAV transmits power to multiple energy receivers simultaneously with high energy transfer efficiency, we design and optimize the 3D energy multi-beams of antenna array mounted on UAV with taking into account mutual coupling affected the

\footnotetext{
${ }^{1}$ The effective aperture is a measure of how effective an received antenna absorbing the power of the transmit antenna, defined as the area which converting the incident power density into collected power [20].
}

beam patterns, which is different from [21], [22]. Furthermore, motivated by [19] and [23], we also consider the influence of UAV's altitude and area coverage on the proposed UAVenabled WPT networks. The main contributions of this paper are summarized as follows.

- We propose a theoretical model for energy harvesting maximization in UAV-enabled WPT networks, where UAV's 3D placement, beam pattern, and charging time are jointly optimized, with constraint of drone flight altitude and wireless coverage performance. However, this energy harvesting optimization problem is non-convex. To tackle the problem, we propose a low-complexity iterative algorithm through sequentially optimizing the UAV's 3D position, beam pattern and charging time.

- First, we adopt sequential unconstrained convex minimization based algorithm [24], [25] to obtain the globally optimal UAV 2D location. Then, based on the horizonal position of UAV and the fixed beam pattern and charging time, the optimal UAV altitude can be obtained easily. Subsequently, we propose the multiobjective evolutionary algorithm based on decomposition (MOEA/D) [26] based algorithm to adjust the antenna gain, side-lobe level (SLL) and beamwidth of energy beamforming by optimizing the phases of array elements. These results show that the MOEA/D based algorithm can approximate the Pareto front. Finally, based on the above solved variables, we use standard convex optimization methods [27] to obtain the optimal charging time.

- We provide numerical results in order to confirm the validity of our theoretical findings and draw design insight into the performance of UAV-enabled WPT networks.

\section{B. Organization and Notation}

The remainder of this paper is organized as follows. In Section II, the UAV-enabled WPT system model is described and the energy harvesting optimization problem is formulated. In Section III, we propose the low-complexity iterative algorithm to solve the energy harvesting optimization problem via sequentially optimizing the UAV's 3D placement, beam pattern and charging time. The branch and bound method is used to design the UAV trajectory in Section IV. Numerical results are presented in Section V to demonstrate the theoretical findings, and finally, conclusions are provided in Section VI.

The following notations are used in this paper. Boldface letters denote vectors. $\mathbf{R}$ denotes a set of real numbers, and $\mathbf{R}_{+}\left(\mathbf{R}_{++}\right)$is the set of nonnegative (strictly positive) real numbers. $\mathbf{a}^{T}$ and $\mathbf{a}^{H}$ are the transpose and complex conjugate transpose of the vector $\mathbf{a}$, respectively. $\|\mathbf{a}\|$ is the Euclidean norm.

\section{System Model AND Problem Formulation}

\section{A. Downlink Channel Model}

We consider the downlink WPT system which consists of a rotary-wing UAV, and $K$ ERs randomly distributed on the ground. The geographical area is divided into $\Gamma$ serving areas according to the distance between ERs. The UAV is mounted 


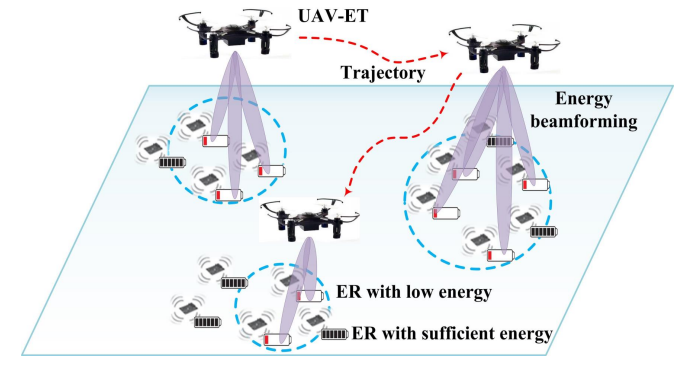

Fig. 1: Illustration of a UAV-enabled WPT with multi-beams.

with an $M \times N$ uniform planar array (UPA) while the ERs have a single antenna. The UAV first hovers at an initial location in one serving area and generates multiple narrow beams to transmit RF signals to ERs simultaneously depending on the location of each ER. For simplicity, we neglect the variability of beam angles due to the wind flow and mechanical vibration of UAV. Subsequently, the UAV will fly to another waypoint according to the trajectory planning ${ }^{2}$. On the other hand, after receiving the RF signals from the energy beamforming, the ERs convert the RF energy and replenish the energy supply via RF-energy harvesting devices. The network architecture is illustrated in Fig. 1. Let ER $k \in\{1,2, \cdots K\}$ have a location on the ground which is denoted by $z_{k}=\left(x_{k}, y_{k}\right)$. The horizontal location of UAV is given by $z_{u}=\left(x_{u}, y_{u}\right)$ and the altitude is $h$. We assume that the wireless channel between the UAV and ER $k$ has the line-of-sight (LOS) path dominated over the non-LOS (NLOS) paths. Thus, the channel $\mathbf{h}_{k}$ between the UAV and the ER $k$ can be expressed as [28], [29]

$$
\mathbf{h}_{k}=\sqrt{\beta_{0} d_{k}^{-\alpha}} \mathbf{a}(\theta, \phi)
$$

where $\alpha(\alpha \geq 2)$ denotes the path loss factor and $\beta_{0}$ is the channel power gain at the reference distance of $d_{0}=1 \mathrm{~m}$. Besides, $d_{k}=\sqrt{\left(x_{k}-x_{u}\right)^{2}+\left(y_{k}-y_{u}\right)^{2}+h^{2}}$ is the distance between the UAV and ER $k . \mathbf{a}(\theta, \phi)$ is the steering vector with the elevation $\theta$ and azimuth $\phi$ angles of the LOS path, which can be defined as

$$
\begin{aligned}
\mathbf{a}(\theta, \phi)= & {\left[1, \cdots, e^{j 2 \pi / \lambda d_{\text {array }} \sin (\theta)[(m-1) \cos (\phi)+(n-1) \sin (\phi)]},\right.} \\
& \left.\cdots, e^{j 2 \pi / \lambda d_{\text {array }} \sin (\theta)[(M-1) \cos (\phi)+(N-1) \sin (\phi)]}\right]^{T},
\end{aligned}
$$

where $\lambda$ and $d_{\text {array }}$ are the wavelength and spacing between antenna elements, respectively. $m$ and $n$ denote the coordinate of antenna elements in $x$ and $y$ directions, respectively. Furthermore, the effective channel gain between UAV and the ER $k$ is given by

$\left|\mathbf{h}_{k}^{H} \mathbf{w}\right|^{2}=\frac{\beta_{0}}{\left[\left(x_{k}-x_{u}\right)^{2}+\left(y_{k}-y_{u}\right)^{2}+h^{2}\right]^{\alpha / 2}}\left|\mathbf{a}^{H}(\theta, \phi) \mathbf{w}\right|^{2}$,

where $\mathbf{w}$ is the beamforming vector which can be linearly adjusted to control the main lobe direction. $\mathbf{E}(\theta, \phi)=\mathbf{a}^{H}(\theta, \phi) \mathbf{w}$ is the synthesized pattern of the $M \times N$ antenna array.

\footnotetext{
${ }^{2}$ We assume that the UAV flies to next waypoint with a constant fligh speed.
}

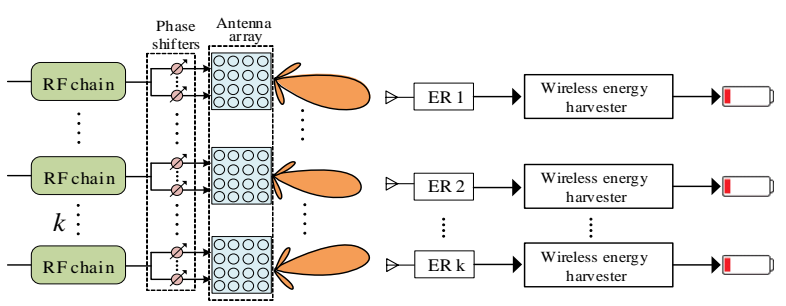

Fig. 2: A transmit and receive structure of analog beamforming based UAV-WPT systems.

\section{B. Transmit and Receive Structure of Analog Beamforming based UAV-enabled WPT Systems}

Different beamforming techniques are available to RF energy transfer. For digital beamforming, each antenna requires a dependent RF chain to control the phase and amplitude of digital signals, resulting in complex hardware architecture and high power consumption. Hence, there are two methods have been proposed to effectively solve this problem. One includes two-stage beamformers, termed hybrid beamforming, which exploits few numbers of RF-chains for digital beamformer while phased shifters are used to perform analog beamformer. However, it needs two-stage feedback (i.e., digital beamforming and analog beamforming), leading to high power consumption for large antenna array. On the other hand, analog beamforming exploits only phase shifters to perform the directional beamforming; thus requires lower hardware complexity and energy consumption [30]. Therefore, in this paper, we adopt the sub-connected analog beamforming technique with multiple RF chains, where each RF chain connects to a subarray. On the other hand, the receivers collect the RF signals through their antenna. The received RF signals are converted to direct current (DC) signals via a wireless energy harvester ${ }^{3}$ to charge the devices with low power, as shown in Fig. 2. We assume that the mutual coupling between two sub-arrays can be ignored, and thus the mutual coupling between antenna elements of a sub-array has been considered in this work. In addition, UAV has the onboard global positioning system (GPS) receiver to estimate geodetic positions. The receivers equipped with a rechargeable battery which can store the harvested energy.

\section{Multiple Steered Beams Generation}

To improve the antenna performance (i.e. antenna gain, beamwidth and side-lobe level) and promote the harvested energy of ERs, antenna arrays are used to generate multiple independent steered beams. In particular, UPA in UAV is separated into several sub-arrays, and each sub-array forms an independent steered beam by adjusting their phase shifters. The direction of beams is determined by ER's local positions which are obtained by the UAV onboard GPS receivers. Thus, for an $M \times N$ antenna array, the array factor and the

\footnotetext{
${ }^{3}$ The model of wireless energy harvester consists of impendence match circuit, voltage multiplier and capacitor [14].
} 


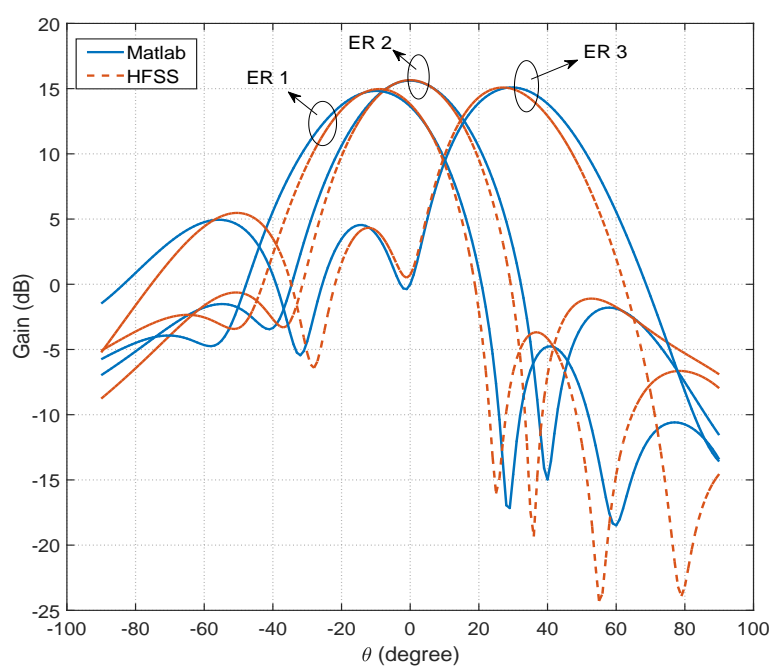

Fig. 3: Antenna array beam pattern of angles $\left(-10^{\circ}, 0^{\circ}\right),\left(0^{\circ}, 0^{\circ}\right)$ and $\left(30^{\circ}, 0^{\circ}\right)$ simulated by MATLAB and HFSS, respectively.

synthesized pattern can be written as [20], [31]

$$
\begin{aligned}
A F & =\sum_{m=1}^{M} \sum_{n=1}^{N} I_{m n} \times e^{j \psi(\theta, \phi)}, \\
\mathbf{E}(\theta, \phi) & =\sum_{m=1}^{M} \sum_{n=1}^{N} p_{m n}(\theta, \phi) I_{m n} \times e^{j \psi(\theta, \phi)},
\end{aligned}
$$

where $\psi(\theta, \phi)=2 \pi / \lambda d_{\text {array }} \sin (\theta)[(m-1) \cos (\phi)+(n-$ $1) \sin (\phi)]+\beta_{m n}, p_{m n}(\theta, \phi)$ and $I_{m n}$ are the active pattern and amplitude excitation of the $(m, n)$-th array element. $\beta_{m n}$ is the progressive phase shift, which can be changed by phase shifters to control the main beam of antenna array towards a particular direction. The beamforming vector $\mathbf{w}=$ $\left[w_{1 n}, \cdots, w_{m n}, \cdots, w_{M N}\right]^{T}$ describes the amplitude excitation and phase of each array element, $w_{m n}=p_{m n}(\theta, \phi) I_{m n}$. $e^{j \beta_{m n}}$. We assume that the amplitude excitation of the entire array is uniform and the spacings between the antenna elements are equal.

Based on the synthesized pattern $\mathbf{E}(\theta, \phi)$, the antenna array divides into $t$ sub-arrays generating three steered beams toward ER 1, ER 2 and ER 3, respectively. The beam patterns of ER 1, ER 2 and ER 3 are shown in Fig. 3, and the angles are $\left(-10^{\circ}\right.$, $\left.0^{\circ}\right),\left(0^{\circ}, 0^{\circ}\right)$ and $\left(30^{\circ}, 0^{\circ}\right)$, respectively ${ }^{4}$. It can be seen in Fig. 3 that the main-lobe gain of all ERs are $7 \mathrm{~dB}$ more than the maximum side-lobe. Besides, it can also be observed that the simulation results using MATLAB can effectively match the one from HFSS.

\section{Energy Harvesting Optimization Problem Formulation}

We assume the energy-storage capacity of each ER is infinite. Thus, the total energy received by each ER $k$ can be written as

$$
Q_{k}=\xi_{k}\left|\mathbf{h}_{k}^{\mathrm{H}} \mathbf{w}\right|^{2} P_{0} \tau_{k, \gamma}
$$

\footnotetext{
${ }^{4}$ In this paper, we assume that the number of serviced ERs is less than the number of sub-arrays. Thus, for the case in Fig. 3, $t \geq 3$.
}

$$
\stackrel{(a)}{=} \frac{\xi_{k} \beta_{0} P_{0} \tau_{k, \gamma}|\mathbf{E}(\theta, \phi)|^{2}}{\left[\left(x_{k}-x_{u}\right)^{2}+\left(y_{k}-y_{u}\right)^{2}+h^{2}\right]^{\alpha / 2}},
$$

where $\xi_{k}\left(0<\xi_{k}<1\right)$ is the energy conversion efficiency which hinges on the hardware circuits of ER $k, P_{0}$ denotes the transmit power of the UAV, $\tau_{k, \gamma}$ denotes the charging time of ER $k$ in the $\gamma$-th serving area. According to $(a)$, we note that the total harvested energy of the ER $k$ is depending on the charging time, beam pattern and the 3D placement of UAV. Thus, we design the optimal policy, $\left\{z_{u}^{*}, h^{*}, \tau_{k, \gamma}^{*}, \mathbf{E}^{*}(\theta, \phi)\right\}$ for maximizing the total energy harvested of all ERs, which can be obtained by solving the following optimization problem

$$
\begin{aligned}
(\mathrm{P} 1): \max _{z_{u}, h, \tau_{k, \gamma}, \mathbf{E}(\theta, \phi)} & \sum_{k=1}^{K} \frac{\xi_{k} \beta_{0} P_{0} \tau_{k, \gamma}|\mathbf{E}(\theta, \phi)|^{2}}{\left[\left\|z_{k}-z_{u}\right\|^{2}+h^{2}\right]^{\alpha / 2}} \\
\text { s.t. } & \left\|z_{k}-z_{u}\right\|^{2} \leq h^{2} \tan ^{2} \Theta, \\
& \sum_{\gamma=1}^{\Gamma} \tau_{k, \gamma}=T, \\
& h_{\text {min }} \leq h \leq h_{\text {max }} .
\end{aligned}
$$

It should be noted that the coverage region of the UAV hovered at altitude $h$ with the effective illumination angle $2 \Theta$ is a circle with radius $h \tan \Theta$ [32]. Constraint (8) specifies the largest horizontal distance between UAV and the ERs that can not exceed the coverage radius of UAV. This constraint guarantees that all the ERs located in the same serving area can be serviced by the UAV simultaneously. Constraint (9) specifies the total charging time of ERs in $\Gamma$ serving areas equals to the whole charging period $T$. In particular, we assume that ERs within the same serving area have the same charging time. Constraint (10) here is used to limit the feasible region of UAV altitude. Since hovering at low altitude may have the safety issues due to the obstacle height, while hovering at high altitude is also not recommended due to authority regulations.

The energy harvesting optimization problem here, which jointly optimizes the UAV's 3D placement, beam pattern and charging time, is mixed-combinatorial and non-convex. The solution of the above problem is therefore nontrivial and cannot be obtained directly. As a result, we develop a joint UAV's 3D placement, beam pattern design and time allocation algorithm to solve the proposed problem by optimizing the above variables sequentially.

\section{JoInt UAV's 3D Placement, BEAM PATtERN Design And Time Allocation Algorithm}

As the energy harvesting optimization problem is nonconvex and difficult to solve it, in this section, we introduce the low-complexity iterative algorithm to solve the energy harvesting optimization problem by decomposing the original problem into four sub-problems and optimizing the UAV's 2D placement $z_{u}$, altitude $h$, beam pattern $\mathbf{E}(\theta, \phi)$ and charging time $\tau_{k, \gamma}$, respectively. Since the beam pattern design depends on the angle of arrival (AOAs) of ERs, it requires to get angle information by determining the UAV's 3D placement. Thus, optimizing the UAV's 3D location first is essential. In particular, the UAV's 2D location should be optimized before the flight height due to the coverage radius is obtained 
when determining horizontal position of a UAV. Based on the acquisition of coverage radius, the UAV altitude can be optimized to guarantee that all serviced ERs are covered. Then, UAV generates the energy beamforming to ERs according to the known angle information. Besides, the sum received power of ERs in different serving areas can be achieved based on the UAV's 3D placement and beam pattern, and optimizing the charging time of ERs can further ensure the fairness of energy transfer among serving areas. Therefore, in our proposed low-complexity iterative algorithm, we first apply the sequential unconstrained convex minimization based algorithm [24] to find the optimal UAV 2D location. Then, with the 2D location of UAV and since the objective function is monotonically decreasing with respect to UAV altitude, we obtain the optimal altitude directly. Subsequently, we propose the multi-beam optimal generation scheme based on MOEA/D [26] to design the steered beam pattern. Finally, we use the standard convex optimization techniques to obtain the globally optimal charging time of ERs.

\section{A. Optimal UAV 2D Location}

To obtain the globally optimal 2D location of UAV, we fix the altitude $h$, beam pattern $\mathbf{E}(\theta, \phi)$ and charging time $\tau_{k, \gamma}$, and then problem (P1) is formulated as

$$
\text { (P2) : } \max _{x_{u}, y_{u}} \sum_{k=1}^{K} \frac{A_{k}}{\left[\left(x_{k}-x_{u}\right)^{2}+\left(y_{k}-y_{u}\right)^{2}+h^{2}\right]^{\alpha / 2}},
$$

where $A_{k}=\xi_{k} \beta_{0} P_{0} \tau_{k, \gamma}|\mathbf{E}(\theta, \phi)|^{2}$ is a constant due to the fixed $h, \mathbf{E}(\theta, \phi)$ and $\tau_{k, \gamma}$. After defining $\varepsilon_{k}\left(x_{u}, y_{u}\right)=$ $\left(x_{k}-x_{u}\right)^{2}+\left(y_{k}-y_{u}\right)^{2}+h^{2}$ and $q_{k}\left(\varepsilon_{k}\left(x_{u}, y_{u}\right)\right)=A_{k}\left[\left(x_{k}-\right.\right.$ $\left.\left.x_{u}\right)^{2}+\left(y_{k}-y_{u}\right)^{2}+h^{2}\right]^{-\alpha / 2}$, problem (P2) can be reformulated as

$$
\text { (P2.1) : } \max _{x_{u}, y_{u}} \sum_{k=1}^{K} q_{k}\left(\varepsilon_{k}\left(x_{u}, y_{u}\right)\right) \text {. }
$$

It should be noted that $\varepsilon_{k}\left(x_{u}, y_{u}\right)$ is a convex function with respect to $\left(x_{u}, y_{u}\right)$, and $q(\cdot)$ is also a strictly convex function with strictly decreasing on $\mathbf{R}_{+}$. It should also be noted that $\lim _{x_{u}, y_{u} \rightarrow \infty} \varepsilon_{k}\left(x_{u}, y_{u}\right)=+\infty$, and $\lim _{\varepsilon_{k}\left(x_{u}, y_{u}\right) \rightarrow \infty} q_{k}\left(\varepsilon_{k}\left(x_{u}, y_{u}\right)\right)=0$. Hence, the function $\sum_{k=1}^{K} q_{k}\left(\varepsilon_{k}\left(x_{u}, y_{u}\right)\right)$ is neither convex nor concave according to convex optimization theory [27]. Nevertheless, the reformulated optimization problem $(\mathrm{P} 2.1)$ can be reformulated to a convex maximization problem, and optimally solved by solving a sequence of unconstrained convex minimization subproblems [24]. In particular, problem (P2.1) can be equivalently expressed in the following problem by introducing an auxiliary variable $\mathbf{t}=\left[t_{1}, t_{2}, \cdots, t_{K}\right]^{T}$

$$
\begin{aligned}
(\mathrm{P} 2.2): \max _{x_{u}, y_{u}, \mathbf{t}} & \sum_{k=1}^{K} q_{k}\left(t_{k}\right) \\
\text { s.t. } & \varepsilon_{k}\left(x_{u}, y_{u}\right) \leq t_{k}, k=1,2, \cdots, K .
\end{aligned}
$$

Denote

$$
\begin{array}{r}
\mathcal{D}=\left\{\mathbf{t} \in \mathbf{R}_{+}^{k}: \varepsilon_{k}\left(x_{u}, y_{u}\right) \leq t_{k}, \quad k=1,2, \cdots, K,\right. \\
\left.\exists\left(x_{u}, y_{u}\right) \in \mathbf{R}^{2}\right\} .
\end{array}
$$

Then, problem (P2.2) can be rewritten as

$$
(\mathrm{P} 2.3): \max _{\mathbf{t} \in \mathcal{D}} q(\mathbf{t}),
$$

where $q(\mathbf{t})=\sum_{k=1}^{K} q_{k}\left(t_{k}\right)$. Since $\mathcal{D}$ is compact convex set and $q(\mathbf{t})$ is a strictly convex function, problem $(\mathrm{P} 2.3)$ is a convex maximization problem [33], and cannot be solved by the standard convex optimization methods. Next, we show that problem $(\mathrm{P} 2.3)$ can be solved by reducing it to solving a sequence of unconstrained convex minimization subproblems.

Similar as [25], we denote

$$
\begin{aligned}
& \mathcal{C}=\left\{\mathbf{t} \in \mathbf{R}_{+}^{k}: q(\mathbf{t}) \leq q\left(\mathbf{t}^{*}\right)\right\}, \\
& \widetilde{\mathcal{D}}=\left\{\mathbf{t}-\mathbf{t}_{0} \mid \mathbf{t} \in \mathcal{D}\right\}, \\
& \widetilde{\mathcal{C}}=\left\{\mathbf{t}-\mathbf{t}_{0} \mid \mathbf{t} \in \mathcal{C}\right\},
\end{aligned}
$$

where $\mathbf{t}^{*}$ is the optimal solution in problem $(\mathrm{P} 2.3), \mathbf{t}_{0} \in \mathcal{D}$ and $q\left(\mathbf{t}_{0}\right)<q\left(\mathbf{t}^{*}\right)$. Since the problem (P2.3) is a convex problem and strictly decreasing with respect to $\mathrm{t}$, the set $\mathcal{C}$ and $\widetilde{\mathcal{C}}$ are convex and closed set, $0 \in \widetilde{\mathcal{D}} \cap$ int $\widetilde{\mathcal{C}}$. Besides, (17) and (19) reveal that the points $\mathbf{t}$ in the set $\mathcal{C}$ and $\widetilde{\mathcal{C}}$ are not better than the current best point $\mathbf{t}^{*}$ since $q(\mathbf{t}) \leq q\left(\mathbf{t}^{*}\right)$, and thus the points in the set $\mathcal{C}$ and $\widetilde{\mathcal{C}}$ are not the global optimal. If $\widetilde{\mathcal{D}} \subset \widetilde{\mathcal{C}}$, all the points in $\widetilde{\mathcal{D}}$ are not the global optimal. In other words, $\mathbf{t}^{*}$ is a globally optimal solution. On the other hand, if $\widetilde{\mathcal{D}} \not \subset \widetilde{\mathcal{C}}$, at least one point $\mathbf{t}$ in $\widetilde{\mathcal{D}}$ may be the globally optimal solution. To determine whether $\widetilde{\mathcal{D}} \subset \widetilde{\mathcal{C}}$ or not, we introduce the following propositions.

Proposition 1 (Theorem 4.1 .3 and Exercise 4.1 [34]): Let $\Phi^{\circ}$ be the polar set for $\Phi$, and is defined as

$$
\Phi^{\mathrm{o}}=\left\{\psi \in \Psi \mid \max _{\psi} \psi^{T} x \leq 1, x \in \Phi, \Psi \subset \mathbf{R}^{n}\right\} .
$$

For the subsets $\{P, G\}$ of $\Psi$, proving $P \subset G$ implies $G^{\circ} \subset$ $P^{\circ} . G^{\mathrm{o}}$ and $P^{\mathrm{o}}$ are the polar sets of $G$ and $P$, respectively.

We define $\widetilde{\mathcal{C}^{\circ}}$ and $\widetilde{\mathcal{D}}^{\mathrm{o}}$ as the polar sets of $\widetilde{\mathcal{C}}$ and $\widetilde{\mathcal{D}}$, respectively, and we give the properties of the set $\widetilde{\mathcal{C}}$ in the following proposition.

Proposition 2: $\widetilde{\mathcal{C}}$ contains the orthant $\mathbf{R}_{+}^{K}$.

Proof: Please refer to [24] and [25] for a proof of Proposition 2.

Based on the Proposition 2, we have $\widetilde{\mathcal{C}}^{\mathrm{o}} \subset \mathbf{R}_{-}^{K}$ [35]. Following Proposition 1 and Proposition 2, we can use the property of polar set to determine whether $\underset{\mathcal{D}}{\widetilde{\mathcal{D}}} \underset{\widetilde{\mathcal{C}}}{\widetilde{\mathcal{C}}}$ or not. To facilitate the determination of whether $\widetilde{\mathcal{D}} \subset \widetilde{\mathcal{C}}$ or not, we introduce a polytope $S$ such that $\widetilde{\mathcal{C}}^{\mathrm{o}} \subset S \subset \mathbf{R}_{-}^{K}$. The following proposition can check whether $\widetilde{\mathcal{D}} \subset \widetilde{\mathcal{C}}$.

Proposition 3: Denote $\mathcal{V}$ as the vertex set of polytope $S$. $\widetilde{\mathcal{C}}^{\mathrm{o}} \subset \widetilde{\mathcal{D}}^{\mathrm{o}}$ holds if and only if

$$
\max _{\mathbf{t} \in \widetilde{\mathcal{D}}} \mathbf{v}^{T} \mathbf{t} \leq 1, \forall \mathbf{v} \in \mathcal{V}
$$

where $\mathbf{v}=\left[v_{1}, \cdots, v_{K}\right]^{T}$.

Proof: $\widetilde{\mathcal{D}}^{\circ}$ is a convex set according to the property of polar set [34], and thus we have $S \subset \widetilde{\mathcal{D}}^{\mathrm{o}}, \widetilde{\mathcal{C}}^{\mathrm{o}} \subset \widetilde{\mathcal{D}}^{\mathrm{o}}$ if and only if $\mathcal{V} \subset \widetilde{\mathcal{D}}^{\circ}$. To guarantee $\mathcal{V} \subset \widetilde{\mathcal{D}}^{\circ}$, we have $\mathbf{v} \in \widetilde{\mathcal{D}}^{\circ}, \forall \mathbf{v} \in \mathcal{V}$. According to Proposition $1, \mathbf{v}$ is the polar set of $\mathbf{t} \in \widetilde{\mathcal{D}}$ and $\mathbf{v} \in \widetilde{\mathcal{D}}^{\mathrm{o}}$ if and only if (21) holds. This completes the proof of the Proposition 2. 
Therefore, when $\widetilde{\mathcal{C}^{\circ}} \subset \widetilde{\mathcal{D}}^{\text {o }}$ holds, we can derive $\widetilde{\mathcal{D}} \subset \widetilde{\mathcal{C}}$ according to Proposition 1. In order to determine whether (21) holds, we solve the following sub-problem

$$
\max _{\mathbf{t} \in \widetilde{\mathcal{D}}} \mathbf{v}^{T} \mathbf{t} .
$$

We set $-\mathbf{v}=\mathbf{w}=\left[w_{1}, \cdots, w_{K}\right]^{T}$ and associate (11) and (18), then problem (22) can be equivalently represented as

$$
\min _{x_{u}, y_{u}} \sum_{k=1}^{K} w_{k}\left[\varepsilon_{k}\left(x_{u}, y_{u}\right)-t_{0, k}\right],
$$

where $\mathbf{t}_{0}=\left[t_{0,1}, \cdots, t_{0, K}\right]^{T}$. Since $-\mathbf{w}^{T} \mathbf{t}_{0}$ is a constant, it has no impact on the objective function (23) and hence it can be reduced. Therefore, problem (23) can be equivalently expressed as

$$
\min _{x_{u}, y_{u}} \sum_{k=1}^{K} w_{k}\left[\left(x_{k}-x_{u}\right)^{2}+\left(y_{k}-y_{u}\right)^{2}+h^{2}\right] .
$$

As a result, we have the following closed-form solution of UAV's 2D location

$$
x_{u}^{*}=\frac{\sum_{k=1}^{K} w_{k} x_{k}}{\sum_{k=1}^{K} w_{k}}, \quad y_{u}^{*}=\frac{\sum_{k=1}^{K} w_{k} y_{k}}{\sum_{k=1}^{K} w_{k}} .
$$

We denote $\mu(\mathbf{w})$ as the optimal objective value of problem (24), then the value of $\mathbf{v}^{T} \mathbf{t}$ in (22) is $-\mu(\mathbf{w})+\mathbf{w}^{T} \mathbf{t}_{0}$. If the value of $\mathbf{v}^{T} \mathbf{t} \leq 1, \mathbf{t}^{*}$ is the global optimum according to Proposition 3. On the other hand, if $\mathbf{v}^{T} \mathbf{t}>1$, there may exist a feasible solution $\widetilde{\mathbf{t}}=\left[\widetilde{t}_{1}, \cdots, \widetilde{t}_{K}\right]^{T}$ better than $\mathbf{t}^{*}$. Then, we can obtain

$$
\widetilde{x}_{u}=\frac{\sum_{k=1}^{K} \widetilde{w}_{k} x_{k}}{\sum_{k=1}^{K} \widetilde{w}_{k}}, \quad \widetilde{y}_{u}=\frac{\sum_{k=1}^{K} \widetilde{w}_{k} y_{k}}{\sum_{k=1}^{K} \widetilde{w}_{k}},
$$

and

$$
\widetilde{t}_{k}=\varepsilon_{k}\left(\widetilde{x}_{u}, \widetilde{y}_{u}\right), k=1, \cdots, K .
$$

Hence, to obtain the complete solution of the global optimal UAV 2D location, we check whether feasible solution $\widetilde{\mathbf{t}}$ better than $\mathbf{t}^{*}$ using the following two cases.

Case 1: $q(\widetilde{\mathbf{t}})>q\left(\mathbf{t}^{*}\right)$ denotes feasible solution $\widetilde{\mathbf{t}}$ is better than the current solution $\mathbf{t}^{*}$.

Case 2: $q(\widetilde{\mathbf{t}}) \leq q\left(\mathbf{t}^{*}\right)$ denotes $\mathbf{t}^{*}$ is the globally optimal solution.

For case 2, we perform the analytic center cutting plane method (ACCPM) [22] to separate the points which are not the global solutions, and the key idea of this algorithm is to determine the cutting planes by computing

$$
\vartheta=\sup \left\{\rho: q\left(\mathbf{t}_{0}+\rho\left(\widetilde{\mathbf{t}}-\mathbf{t}_{0}\right)\right) \leq q\left(\mathbf{t}^{*}\right)\right\},
$$

and $\vartheta \geq 1$ due to $q\left(\mathbf{t}_{0}\right)<q\left(\mathbf{t}^{*}\right)$ and $q(\widetilde{\mathbf{t}}) \leq q\left(\mathbf{t}^{*}\right)$. Based on $\vartheta$, we have the following cutting plane.

Proposition 4: The cutting plane

$$
\mathbf{t}^{T}\left(\widetilde{\mathbf{t}}-\mathbf{t}_{0}\right) \leq \frac{1}{\vartheta}
$$

excludes $\mathbf{t}=-\widetilde{\mathbf{w}}$ from the polytope $S$ without eliminating any point of $\widetilde{\mathcal{C}^{\circ}}$.
Proof: Setting $\mathbf{t}=-\widetilde{\mathbf{w}}$, we can obtain

$$
\begin{aligned}
\mathbf{t}^{T}\left(\widetilde{\mathbf{t}}-\mathbf{t}_{0}\right) & =-\widetilde{\mathbf{w}}^{T} \widetilde{\mathbf{t}}+\widetilde{\mathbf{w}}^{T} \mathbf{t}_{0} \\
& =-\mu(\widetilde{\mathbf{w}})+\widetilde{\mathbf{w}}^{T} \mathbf{t}_{0}>1 .
\end{aligned}
$$

Thus, $\mathbf{t}=-\widetilde{\mathbf{w}}$ violates the cutting plane (29). Besides, according to (29), we have $\mathbf{t}_{0}+\vartheta\left(\widetilde{\mathbf{t}}-\mathbf{t}_{0}\right) \in \mathcal{C}$ according to the definition of $\mathcal{C}$, while $\vartheta\left(\widetilde{\mathbf{t}}-\mathbf{t}_{0}\right) \in \widetilde{\mathcal{C}}$ according to the definition of $\widetilde{\mathcal{C}}$. Then, we have

$$
\mathbf{t}^{T}\left(\widetilde{\mathbf{t}}-\mathbf{t}_{0}\right) \leq \frac{1}{\vartheta}, \forall \mathbf{t} \in \widetilde{\mathcal{C}}^{\mathrm{o}} .
$$

From (31), any point $\mathbf{t} \in \widetilde{\mathcal{C}}^{\text {o }}$ satisfies (29). This completes the proof of Proposition 4.

Based on the cutting plane (29), we use the ACCPM to obtain a sequential nested polytope $S$, and the $(m+1)$-th polytope can be given by

$$
S^{(m+1)}=S^{(m)} \cap\left\{\mathbf{t}: \mathbf{t}^{T}\left(\widetilde{\mathbf{t}}-\mathbf{t}_{0}\right) \leq \frac{1}{\vartheta}\right\} .
$$

Next, we should construct $S^{(1)}$ of (32) which satisfies Proposition 4, i.e, $\widetilde{\mathcal{C}}^{\mathrm{o}} \subset S^{(1)}$. We can always select $\varsigma>0$ small enough such that $-\varsigma \mathbf{e} \in \widetilde{\mathcal{C}}$ due to $0 \in$ int $\widetilde{\mathcal{C}}$, where $\mathbf{e}=[1, \cdots, 1]^{T}$ denotes a vector of ones in $\mathbf{R}^{K}$. Hence, we have the following proposition to construct $S^{(1)}$.

Proposition 5: If

$$
S^{(1)}=\left\{\mathbf{t} \in \mathbf{R}_{-}^{K}:-\sum_{k=1}^{K} t_{k} \leq \frac{1}{\varsigma}\right\},
$$

we can obtain $\widetilde{\mathcal{C}^{\circ}} \subset S^{(1)}$.

Proof: According to the definition of polar set, we have $\mathbf{t}^{T}(-\varsigma \mathbf{e}) \leq 1$ for all $\mathbf{t} \in \widetilde{\mathcal{C}}^{\mathrm{o}}$. Then, we have

$$
-\sum_{k=1}^{K} t_{k} \leq \frac{1}{\varsigma}, \forall \mathbf{t} \in \widetilde{\mathcal{C}}^{o} .
$$

Therefore, $\widetilde{\mathcal{C}^{\circ}} \subset S^{(1)}$ holds.

Hence, starting from $S^{(1)}$, a sequence of nested polytopes in $\mathbf{R}_{-}^{K}$ can be constructed as

$$
S^{(1)} \supset S^{(2)} \supset \cdots \supset S^{(m)} \supset \cdots \widetilde{\mathcal{C}}^{\circ} .
$$

Next, we introduce the sequential unconstrained convex minimization based algorithm to find the optimal UAV 2D location, and the whole algorithm procedure is summarized in TABLE I. In the $m$-th iteration, if all the $-\mathbf{w} \in \mathcal{V}^{(m)}$ satisfies $\max _{-\mathbf{w} \in \mathcal{V}^{(m)}}-\mu(\mathbf{w})+\mathbf{w}^{T} \mathbf{t}_{0} \leq 1$, then $\mathbf{t}^{*}$ is the global optimal solution. Otherwise, computing $\widetilde{\mathbf{t}}$, if $q(\widetilde{\mathbf{t}})>q\left(\mathbf{t}^{*}\right)$, we then update $\mathbf{t}^{*}$. On the other hand, if $q(\widetilde{\mathbf{t}}) \leq q\left(\mathbf{t}^{*}\right)$, we eliminate the points which are not the global optimal solutions using the cutting plane $S^{(m+1)}$.

Remark 1: The vertex set of $S^{(1)}$ is $\mathbf{v}^{(1)}=\left\{-\frac{1}{\varsigma} \mathbf{e}_{k} \in \mathbf{R}^{K}\right.$ : $1 \leq k \leq K\} \cup\{0\}$, and we use the Multi-Parametric Toolbox 3.0 [36] to solve the vertex enumeration problem. In addition, the convergence analysis of the algorithm is similar to that in [24], and thus is omitted for brevity. 
TABLE I: THE SEQUENTIAL UNCONSTRAINED CONVEX MINIMIZATION BASED ALGORITHM

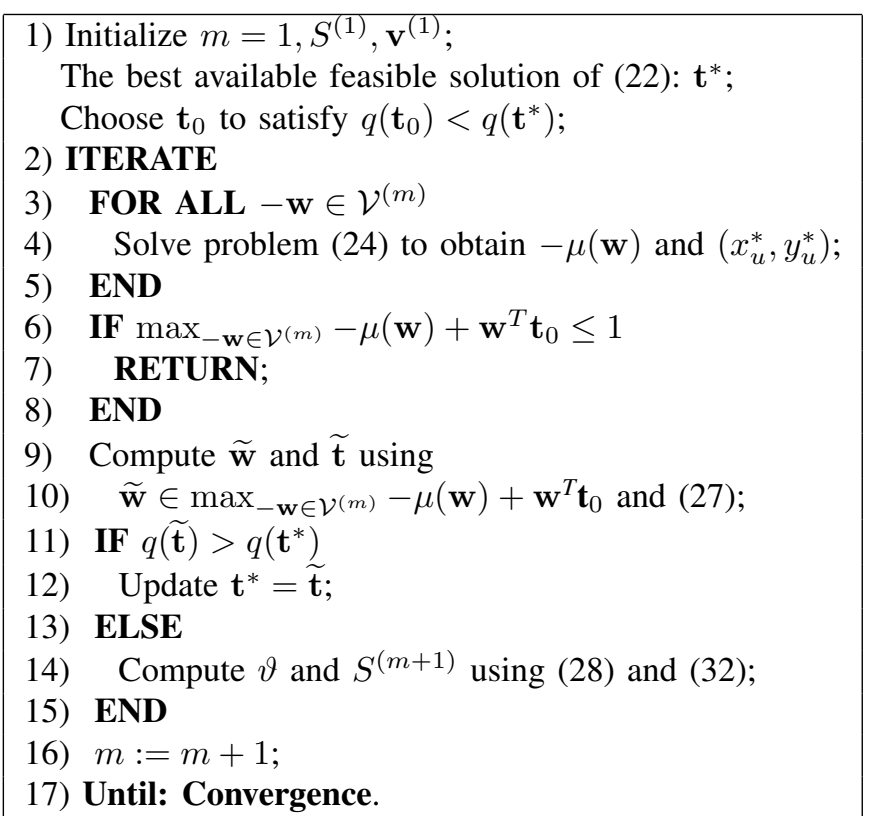

\section{B. Optimal UAV Altitude}

Based on the solved 2D location of UAV, we are now optimizing the UAV altitude. Specifically, we fix $\mathbf{E}(\theta, \phi)$ and $\tau_{k, \gamma}$, and the problem (P1) can be formulated as

$$
\begin{aligned}
\text { (P3) : } \max _{h} & \sum_{k=1}^{K} \frac{A_{k}}{\left[D_{k}+h^{2}\right]^{\alpha / 2}} \\
\text { s.t. } & D_{\max } \leq h^{2} \tan ^{2} \Theta, \\
& h_{\min } \leq h \leq h_{\max },
\end{aligned}
$$

where $D_{k}=\left\|z_{k}-z_{u}\right\|^{2}, D_{\max }=\max _{k=1, \cdots, K} D_{k}$. Constraint (37) specifies the largest horizontal distance between the UAV and ERs can not exceed the coverage radius of the UAV. This constraint guarantees that all ERs are within wireless coverage area of the UAV. It is noted that the objective function of problem (P3) is monotonic decreasing with respect to $h$. Thus, we can easily obtain the optimal solution as

$$
h^{*}=\max \left\{\frac{\sqrt{D_{\max }}}{\tan \Theta}, h_{\min }\right\} .
$$

The physical meaning of (39) is to obtain the minimum valid altitude which guarantees that all the ERs are within the UAV's maximum wireless coverage, and thus improves the energy efficiency of UAV with limited transmit power.

\section{Optimal Phased-Array Pattern}

After obtaining the 3D position of UAV, we then design the parameters of antenna array (the SLL, antenna gain and beamwidth) to generate steered beam pattern to a desired position with fixed charging time $\tau_{k, \gamma}$. Hence, problem (P1) can be reformulated as

$$
\text { (P4) : } \max \sum_{k=1}^{K} \eta_{k}\left|\mathbf{E}_{k}(\theta, \phi)\right|^{2},
$$

where $\eta_{k}=\frac{\xi_{k} \beta_{0} P_{0} \tau_{k, \gamma}}{\left[\left\|z_{k}-z_{u}\right\|^{2}+h^{2}\right]^{\alpha / 2}}$ and is constant for ER $k$. As mentioned earlier, the $M \times N$ antenna array is divided into several sub-arrays with ignoring the effect of mutual coupling between sub-arrays, and each sub-array creates a steerable beam. This implies that the beams $\mathbf{E}_{k}(\theta, \phi)(k \in\{1, \cdots, K\})$ generated by sub-arrays are essentially independent of each other. Thus, problem (P4) can be equivalently expressed as problem (P4.1) as follows

$$
\text { (P4.1) : } \max \mathbf{E}_{k}(\theta, \phi) .
$$

In particular, to form a directional beam pattern, the key idea is controlling the phase of antenna elements to adjust these array parameters, which can be constructed as a multiobjective optimization problem. To tackle this problem, we propose the MOEA/D based algorithm to solve it via iteration process. Thus, to facilitate the introduction of MOEA/D based algorith$\mathrm{m}$, we first provide a definition and a proposition as follows.

Definition 1 ([26]): A multiobjective optimization problem (MOP) can be defined as

$$
\begin{aligned}
\max & F(\mathbf{x})=\left(f_{1}(\mathbf{x}), \cdots, f_{m}(\mathbf{x})\right)^{T} \\
\text { s.t. } & \mathbf{x} \in \Omega,
\end{aligned}
$$

where $\mathbf{x}=\left(x_{1}, x_{2}, \cdots, x_{n}\right)^{T}$ denotes decision variables while $\Omega=\left\{\mathbf{x} \in \mathbf{R}^{n} \mid h_{j}(\mathbf{x}) \leq 0, j=1, \cdots, m\right\}$ is decision space. $h_{j}(\mathbf{x})$ are continuous functions. Based on the definition of MOP, we construct the beam pattern synthesis problem ${ }^{5}$ as a multiobjective optimization problem with respect to phases $\boldsymbol{\beta}$ in the following.

Proposition 6: Optimizing the phase of $M \times N$ antenna array elements to adjust the antenna gain, SLL and beamwidth simultaneously, which can be modelled as a multiobjective optimization problem

$$
\begin{aligned}
& \min F(\boldsymbol{\beta})=\left(f_{1}(\boldsymbol{\beta}), f_{2}(\boldsymbol{\beta}), f_{3}(\boldsymbol{\beta})\right)^{T} \\
& \text { s.t. } \boldsymbol{\beta} \in \mathbf{R}^{M \times N},
\end{aligned}
$$

where $f_{1}(\boldsymbol{\beta})=S L L(\boldsymbol{\beta}), f_{2}(\boldsymbol{\beta})=\frac{1}{|\mathbf{E}(\theta, \phi)|}, f_{3}(\boldsymbol{\beta})=\frac{1}{\left|\Theta_{h, e}\right|}$, $\boldsymbol{\beta}=\left[\beta_{1 n}, \cdots, \beta_{m n}, \cdots, \beta_{M N}\right]^{T}$ and $\beta_{m n}=(m-1) \beta_{x}+$ $(n-1) \beta_{y}$.

Proof: Please refer to Appendix A for a proof of Proposition 6.

Next, we present the MOEA/D based algorithm to solve problem (44)-(45), and the steps are summarized in TABLE II. The proposed algorithm applies Tchebycheff approach [26] to decompose the multiobjective optimization problem into a number of scalar optimization problems, and then optimize all the subproblems simultaneously to approximate the Pareto front by exploiting their relationship. Specifically, in step Initiation, we first use the Euclidean distance to compute the closest $T_{n e i}$ weight vectors of $\boldsymbol{\kappa}^{i}\left(\boldsymbol{\kappa}^{i}=\left(\kappa_{1}^{i}, \cdots, \kappa_{d}^{i}\right)^{T}\right)$ in the

\footnotetext{
${ }^{5}$ The beam synthesis problem is a problem that designing the number of array elements, spacing, excitation amplitude and phase according to the radiation characteristics, such as beamshape, beamwidth, SLL and directivity.
} 
TABLE II: MOEA/D BASED ALGORITHM

\section{1) INPUT}

Model MOP as (44)-(45);

iter: the number of iterations;

$N_{\text {pop }}$ : the number of subproblems in MOEA/D;

$\boldsymbol{\kappa}^{1}, \cdots, \boldsymbol{\kappa}^{N_{p o p}}$ : weight vectors;

$T_{n e i}$ : the number of the weight vectors in the

neighborhood of each weight vector;

2) OUTPUT

3) EP: non-dominated solutions during the search;

4) INITIATION

5) Set $\mathrm{EP}=\emptyset$;

6) FOR each $i=1, \cdots, N_{\text {pop }}$, compute $T_{n e i}$ closest weight vectors $\boldsymbol{\kappa}^{i_{1}}, \cdots, \boldsymbol{\kappa}^{i_{T_{n e i}}}$ to weight vector $\boldsymbol{\kappa}^{i}$, set $\hbar(i)=\left\{i_{1}, \cdots, i_{T_{n e i}}\right\}$;

7) Generate an initiate population $\boldsymbol{\beta}_{1}, \cdots, \boldsymbol{\beta}_{N_{p o p}}$ randomly. Set $F V_{i}=F\left(\boldsymbol{\beta}_{i}\right)$;

8) Initiate $\mathbf{z}=\left(z_{1}, \cdots, z_{j}, \cdots, z_{d}\right)^{T}$ by $z_{j}=\min \left\{f_{j}(\boldsymbol{\beta}), \boldsymbol{\beta} \in \mathbf{R}^{M \times N}\right\}$

9) UPDATE

10) FOR $i=1, \cdots, N_{\text {pop }}$

11) Randomly select two indexes $k, l$ from $\hbar(i)$, and generate a new solution $\boldsymbol{y}$ from $\boldsymbol{\beta}_{k}$ and $\boldsymbol{\beta}_{l}$;

12) $\quad$ FOR each $j=1, \cdots, d$, set $z_{j}=f_{j}(\boldsymbol{y})$, IF $z_{j}>f_{j}(\boldsymbol{y})$

13) FOR each $j \in \hbar(i)$, set $\boldsymbol{\beta}_{j}=\boldsymbol{y}$ and $F V_{j}=F(\boldsymbol{y})$, $\mathbf{I F} g^{t e}\left(\boldsymbol{y} \mid \boldsymbol{\kappa}^{j}, \mathbf{z}\right) \leq g^{t e}\left(\boldsymbol{\beta}_{j} \mid \boldsymbol{\kappa}^{j}, \mathbf{z}\right)$;

14) Remove all vectors dominated by $F(\boldsymbol{y})$ from EP;

15) Add $F(\boldsymbol{y})$ to EP IF no vectors dominate $F(\boldsymbol{y})$;

16) STOPPING CRITERIA

17) Iterates iter times, stop and output EP. OTHERWISE, go to UPDATE.

neighborhood for each $i \in\left\{1, \cdots, N_{\text {pop }}\right\}$, and the indexes of $T_{n e i}$ contains in $\hbar(i)$. Then, according to the number of subproblems, we generate the decision variables (progressive phase shift $\boldsymbol{\beta}$ ) randomly. $\mathbf{z}=\left(z_{1}, \cdots, z_{j}, \cdots, z_{d}\right)^{T}$ denotes the best-so-far solution for the objective function $f_{j}(\boldsymbol{\beta})$ and $F V_{i}$ is the F-value of $\boldsymbol{\beta}_{i}$. In step Update, for each subproblem $i$, we randomly select two indexes $k$ and $l$ from $\hbar(i)$, and then generate a new decision variable $\boldsymbol{y}$ from $\boldsymbol{\beta}_{k}$ and $\boldsymbol{\beta}_{l}$ by differential evolution (DE) algorithm [37]. If $z_{j}>f_{j}(\boldsymbol{y})$, for each $j=1, \cdots, d$, we update the reference point $z_{j}$. Then, for each $j \in \hbar(i)$, if $g^{t e}\left(\boldsymbol{y} \mid \boldsymbol{\kappa}^{j}, \mathbf{z}\right) \leq g^{t e}\left(\boldsymbol{\beta}_{j} \mid \boldsymbol{\kappa}^{j}, \mathbf{z}\right)$, we replace the decision variable $\boldsymbol{\beta}_{j}$ and $F$-value $F V_{j}$ by $\boldsymbol{y}$ and $F(\boldsymbol{y})$, respectively, where $g^{t e}\left(\boldsymbol{y} \mid \boldsymbol{\kappa}^{j}, \mathbf{z}\right)=\max _{1 \leq t \leq d}\left\{\kappa_{t}^{j}\left|f_{t}(\boldsymbol{y})-z_{t}\right|\right\}$ [26]. If the number of iterations is iter, stop and output EP. Otherwise, go to the step Update. Finally, the convergence to the Pareto optimal front of the proposed MOEA/D based algorithm has been proven. We first give the following definitions.

Definition 2 ( [26]): Assuming $u, v \in \mathbf{R}^{m}, u$ dominates $v^{6}$ if and only if

$$
u_{i} \leq v_{i}, i \in\{1, \cdots, m\}
$$

${ }^{6}$ This definition of domination is in the case of minimization. and

$$
u_{j}<v_{j}, \exists j \in\{1, \cdots, m\} .
$$

Definition 3 ([26]): A point $\mathrm{x}^{*} \in \Omega$ is Pareto optimal which satisfies no point $\mathbf{x} \in \Omega$ such that $F(\mathbf{x})$ dominated $F\left(\mathbf{x}^{*}\right)$, and $F\left(\mathbf{x}^{*}\right)$ is a Pareto optimal (objective) vector.

Based on the above definitions, we can obtain the Pareto set of problem (44)-(45) as follows.

Proposition 7: The multiobjective optimization problem (44)-(45) can obtain the Pareto optimal solutions, and the Pareto set is

$$
\begin{aligned}
P^{*}= & \left\{\boldsymbol{\beta}^{*}\right\} \\
= & \left\{\boldsymbol{\beta} \in \mathbf{R}^{M \times N} \mid \neg \exists \boldsymbol{\beta}^{\prime} \in \mathbf{R}^{M \times N}, f_{i}\left(\boldsymbol{\beta}^{\prime}\right) \leq f_{i}\left(\boldsymbol{\beta}^{*}\right)\right\}, \\
& i \in 1, \cdots, 3 .
\end{aligned}
$$

Proof: Please refer to Appendix B for a proof of Proposition 7.

Proposition 7 indicates that MOEA/D based algorithm used to generate the beam patterns can converge to the Pareto optimal set. Next, based on the solved variables, we optimize the charging time using the standard convex optimization techniques.

\section{Optimal Charging Time}

Based on the solved UAV 3D placement and the beam pattern, we finally optimize the charging time of ER $k$ in $\gamma$-th serving area. Thus, problem (P1) can be simplified as

$$
\begin{aligned}
(\mathrm{P} 5): \max _{\tau_{k, \gamma}} & \sum_{k=1}^{K} \frac{\xi_{k} \beta_{0} P_{0} \tau_{k, \gamma}|\mathbf{E}(\theta, \phi)|^{2}}{\left[\left\|z_{k}-z_{u}\right\|^{2}+h^{2}\right]^{\alpha / 2}} \\
\text { s.t. } & \sum_{\gamma=1}^{\Gamma} \tau_{k, \gamma}=T .
\end{aligned}
$$

The solution of problem (P5) may lead to a severe fairness issue between serving areas. In particular, the sum harvested energy of ERs in one serving area is larger than the sum harvested energy of ERs in other serving areas, where ERs in this region can be charged for a much longer time. Thus, to overcome this issue, we consider a new optimization problem which maximizes the minimum sum received energy among all serving areas, and this formulation can be written as

$$
\begin{aligned}
(\mathrm{P} 5.1): \max _{\tau_{k, \gamma}} & \sum_{k=1}^{K} \min _{k \in K} \frac{\xi_{k} \beta_{0} P_{0} \tau_{k, \gamma}|\mathbf{E}(\theta, \phi)|^{2}}{\left[\left(x_{k}-x_{u, \gamma}\right)^{2}+\left(y_{k}-y_{u, \gamma}\right)^{2}+h_{\gamma}^{2}\right]^{\alpha / 2}} \\
\text { s.t. } & \sum_{\gamma=1}^{\Gamma} \tau_{k, \gamma}=T
\end{aligned}
$$

It should be noted that the UAV's 3D placement in the $\gamma$ th serving area is $\left(x_{u, \gamma}, y_{u, \gamma}, h_{\gamma}\right)$. To solve problem (P5.1), we introduce an auxiliary variable $t$ and problem (P5.1) can be equivalently expressed as

$$
(\mathrm{P} 5.2): \max _{\tau_{k, \gamma}, t} t
$$




$$
\begin{aligned}
\text { s.t. } & \sum_{\gamma=1}^{\Gamma} \tau_{k, \gamma} \mathcal{Q}\left(x_{u, \gamma}, y_{u, \gamma}, h_{\gamma}\right) \geq t \\
& \sum_{\gamma=1}^{\Gamma} \tau_{k, \gamma}=T
\end{aligned}
$$

and $\mathcal{Q}\left(x_{u, \gamma}, y_{u, \gamma}, h_{\gamma}\right)=\sum_{k=1}^{K} \frac{\xi_{k} \beta_{0} P_{0}|\mathbf{E}(\theta, \phi)|^{2}}{\left[\left(x_{k}-x_{u, \gamma}\right)^{2}+\left(y_{k}-y_{u, \gamma}\right)^{2}+h_{\gamma}^{2}\right]^{\alpha / 2}}$. Note that problem (P5.2) is a linear programming problem, and hence it can be solved directly by standard convex optimization techniques [27].

\section{BRANCH AND BOUND BASED TRAJECTORY DESIGN}

In section III, we optimize 3D hovering location, beam pattern and charging time in $\Gamma$ serving areas. In this section, we design the hover-and-fly trajectory based on the 3D hovering locations. The key idea of the hover-and-fly trajectory is to minimize the total flight distance when visiting all $\Gamma$ serving areas. To solve this problem, the authors in [16] and [17] proposed that the minimum total flying distance could be transformed into the traveling salesman problem (TSP). However, the standard TSP should return to the original point whilst our problem does not need to return to the start. Thus, to transform the flying planning problem into the standard TSP, we add a virtual hovering location as the start point 0 whose distances to all $\Gamma$ real hovering locations are zero. After obtaining the optimal trajectory using branch and bound method, we drop the two edges associated with the dummy locations (i.e. from 0 -th to $j$-th and $i$-th to 0 -th locations, $i \neq 0, j \neq 0$ ), and thus the optimal flying trajectory is designed. Therefore, the minimum total flight distance can be determined using the following optimization problem

$$
\begin{aligned}
(\mathrm{P} 6): \min & \sum_{\substack{i=0, i \neq j}}^{\Gamma} \sum_{\substack{j=0, j \neq i}}^{\Gamma} d_{i j} c_{i j} \\
\text { s.t. } & \sum_{\substack{i=0, i \neq j}}^{\Gamma} c_{i j}=1, \\
& \sum_{\substack{j=0, j \neq i}}^{\Gamma} c_{i j}=1, \\
& \sum_{\substack{i=1 \\
\Gamma}} c_{i 0}=1, \\
& \mu_{i}-\mu_{j}+\delta c_{i j} \leq \delta-1,
\end{aligned}
$$

where $d_{i j}=\sqrt{\left(x_{i}-x_{j}\right)^{2}+\left(y_{i}-y_{j}\right)^{2}+\left(h_{i}-h_{j}\right)^{2}}$ denotes the distance between the $i$ th hovering location $\left(x_{i}, y_{i}, h_{i}\right)$ and the $j$ th hovering location $\left(x_{j}, y_{j}, h_{j}\right)$, and $c_{i j} \in\{0,1\}$. The UAV proceeds from location $i$ to location $j$ if and only if $c_{i j}=1$, otherwise, $c_{i j}=0$. Constraint (58) and (59) specify that each hovering location (other than the start point 0) is visited exactly once. Constraint (60) denotes the number of returns to the original hovering location 0 is 1 . In other words, the UAV departs from the start hovering location 0 , then visits $\Gamma$ hovering locations and finally return to location 0 . The constraint (61) guarantees the number of

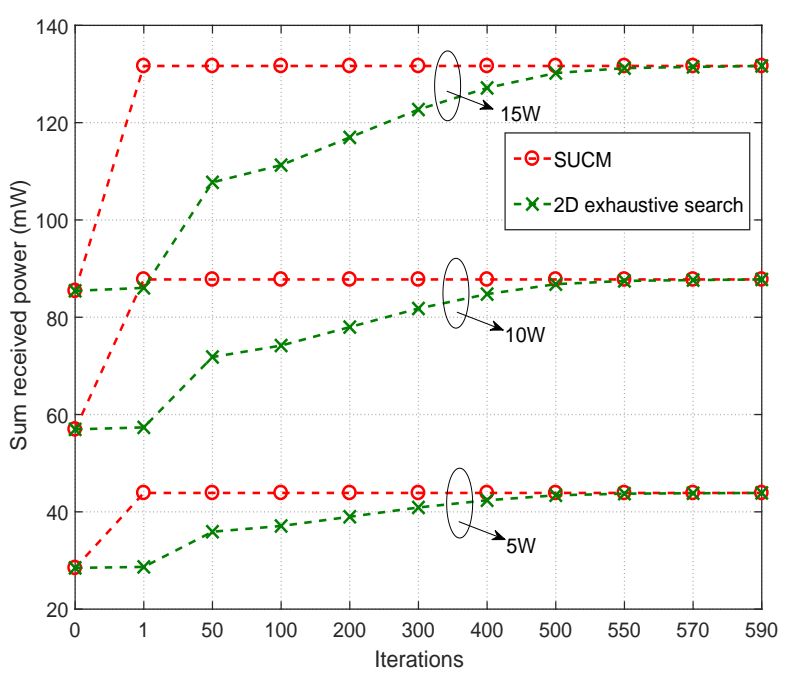

Fig. 4: Convergence behavior of the SUCM and 2D exhaustive search scheme in terms of sum received power.

visited hovering locations should not exceed $\delta(\delta \geq \Gamma)$, where $\mu_{i}(i=1, \cdots, \Gamma)$ are arbitrary real numbers. Problem (P6) is an integer programming problem [38], and thus we use the well-known branch and bound algorithm [39] to obtain the optimal trajectory. In particular, the algorithm first breaks up the set of all trajectories (feasible solutions) into increasingly small subsets and calculates the lower bound on the cost of each subset. Then, the lower bound of a subset which is no greater than all other subsets will contain in the optimal trajectory. Finally, the bounds guide and identify an optimal trajectory.

\section{Simulation Results}

In this section, we present numerical results to evaluate the performance of our proposed solution to the total energy harvested maximization problem (P1). Since millimeter-ware (mm-Ware) communication can provide a high capacity than traditional low-frequency mobile communications, the mmWave channel [28] [29] between UAV and each ER $k$ is adopted with a carrier frequency of $25 \mathrm{GHz}$. We assume that the minimum altitude $h_{\min }$ and maximum altitude $h_{\max }$ are $21 \mathrm{~m}$ and $120 \mathrm{~m}$ respectively [23]. The transmit power $P_{0}$ of the UAV is $10 \mathrm{~W}$, and the charging period is $T=20 \mathrm{~s}$. The energy conversion efficiency $\xi_{k}$ of ER $k$ is set to 0.5 , and the path loss factor $\alpha$ is 2 [25]. The UAV is equipped with $M \times N$ antenna array, and the maximum coverage radius is $h \tan \Theta$. We also assume that there are $K$ ERs with single antenna which are distributed in a $500 \times 500 \mathrm{~m}^{2}$ square area, and are classified into $\Gamma$ groups according to the distance.

In the first simulation, the performance of the proposed solution for the energy harvesting maximization problem is studied. The convergence behavior of the sequential unconstrained convex minimization based algorithm is evaluated by illustration how the sum received power behaves with the number of iterations. To show the global convergence property of this algorithm, we exploit the 2D exhaustive search method for comparison. For convenience, we denote the 


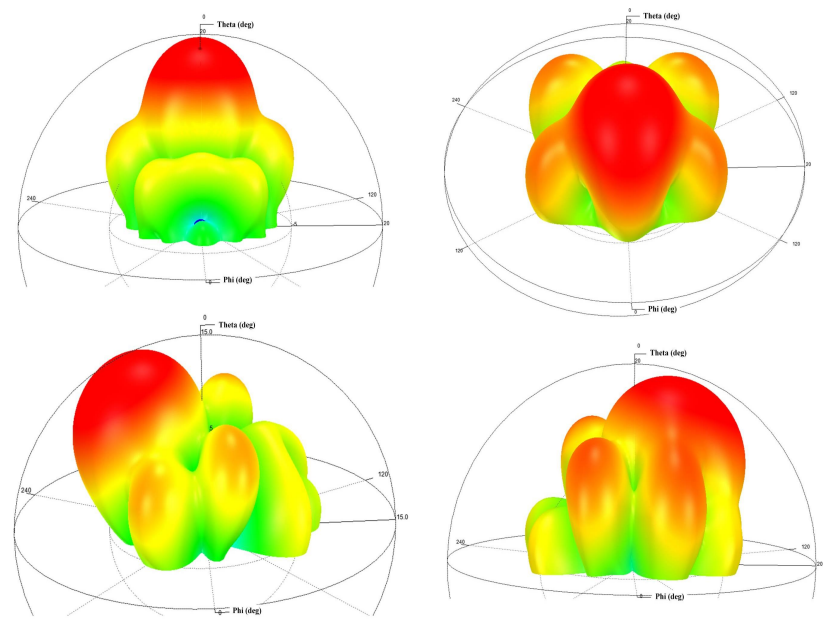

(a)
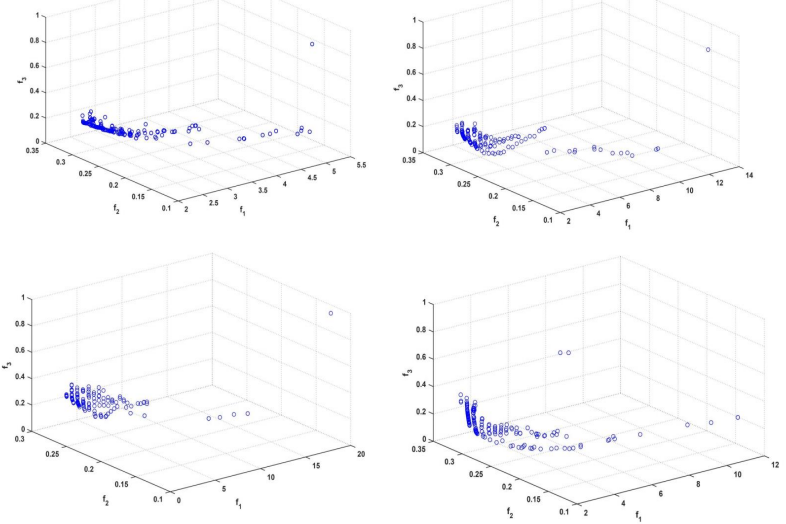

(b)

Fig. 5: Multi-beam gain performance using analog beamforming with MOEA/D based solution (a) 3D realized gain patterns of antenna array. (b) Plot of the non-dominated front by MOEA/D for 3-objective optimization problem.

sequential unconstrained convex minimization based algorithm as SUCM. We assume that there are $K=2$ energy receivers existing in the coverage area. On the other hand, we set the UAV altitude to $h=100 \mathrm{~m}$. As it can be observed in Fig. 4 that the proposed SCUM converges after 1 iteration. Besides, it can be seen that the sum received power increases with increasing transmit power. This is attributed to the fact that a higher transmit power increases the harvested DC power of ERs. Furthermore, as it can be observed that the 2D exhaustive search scheme requires about 590 iterations to converge. More importantly, it can also be observed that the simulation results of the proposed SCUM method match the 2D exhaustive search scheme, but with much faster convergence speed. This demonstrate that the proposed algorithm can converge to a global optimal solution.

We then investigate the antenna array beam pattern synthesis using MOEA/D based algorithm. The UAV is equipped with an $8 \times 8$ antenna array which is divided into four $4 \times 4$ sub-arrays.

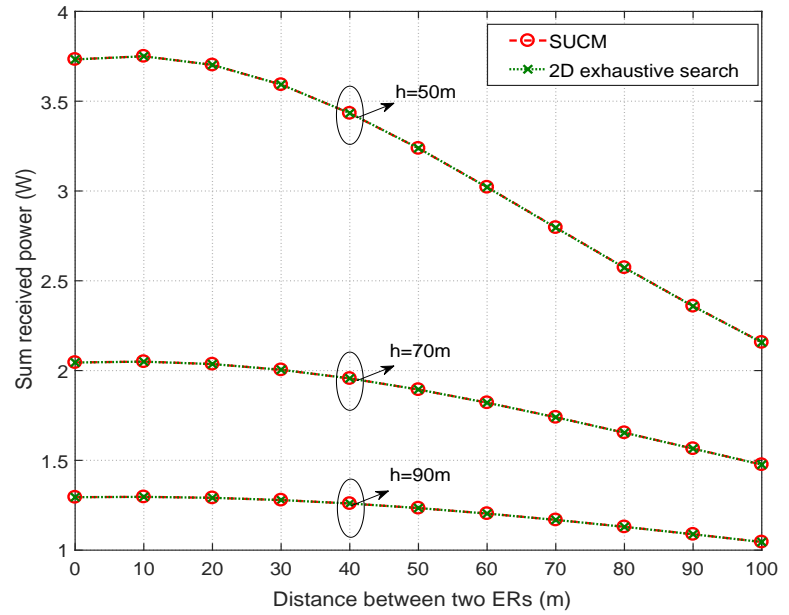

Fig. 6: The performance of two algorithm with different UAV altitudes (sum received power versus the distance between the two ERs).

We assume that the amplitude and spacing of antenna array are $1 \mathrm{~A}$ and $5.5 \mathrm{~mm}$, respectively. Besides, the maximum effective illumination angle $2 \Theta$ is set to $80^{\circ}$. Fig. 5(a) illustrates the scanning capability in angles of $\left(-10^{\circ}, 0^{\circ}\right),\left(30^{\circ}, 0^{\circ}\right),\left(30^{\circ}\right.$, $\left.270^{\circ}\right)$ and $\left(20^{\circ}, 90^{\circ}\right)$. In particular, we first use the MOEA/D based algorithm to optimize the phases of antenna array and obtain the Pareto optimal set. Then, we choose a set of phases and input them into the HFSS to generate the 3D realized gain pattern towards the above angles. We can observe from Fig. 5(a) that four scanned beams having various SLL, beamwidth and antenna gain with different design parameters (i.e. phases $\boldsymbol{\beta})$. This is because that the interference of mutual coupling between antenna elements can be changed by the amplitude excitation and phase set for each antenna, and thus affects the beam pattern. On the other hand, Fig. 5(b) shows the non-dominated set of the above illustrated angles which are obtained after 300 iterations. It can be observed that most of the optimal solutions converge to a real Pareto front. It should be noted that choosing different phases of antenna array leads to different SLL, antenna gain and beamwidth of beam pattern, and thus needs the decision-maker to select the set of phases according to their preference. For instance, the beams with high antenna gain have typically high SLL and wide beamwidth. Therefore, there is a tradeoff between antenna gain, SLL and beamwidth. In addition, if we increase the number of iterations, all the non-dominated solutions will converge to a Pareto front. This is due to the fact that the new non-dominated solution set is closer to the Pareto optimal solution than the old one. After infinite iterations, the nondominated solution will achieve to the Pareto optimal set.

In the next simulation, the sum received power under different constraints are evaluated and presented in Fig. 6-Fig. 7. The sum received power under different distance between two ERs are evaluated and presented first. We assume that $K=2$ energy receivers are distributed in a serving area. In Fig. 6, it is expected that the sum received power is monotonically non-increasing with respect to the distance between two ERs. 


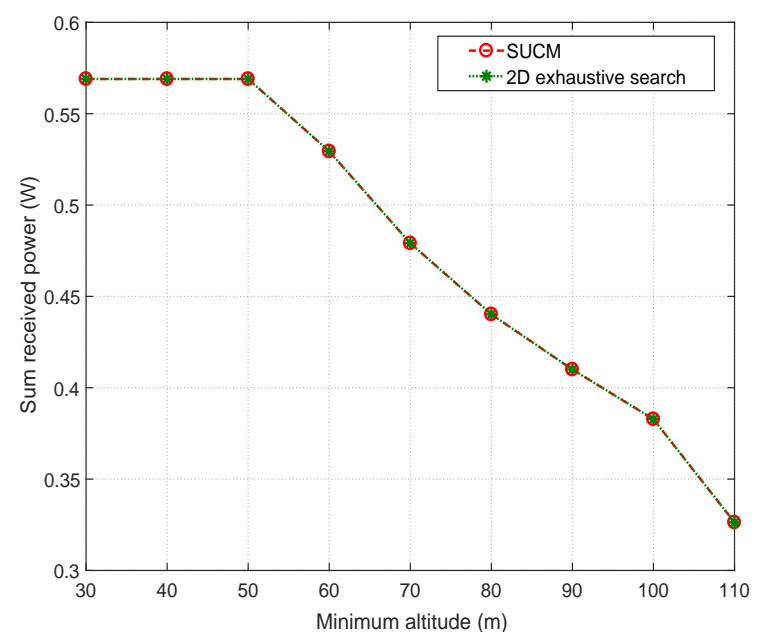

Fig. 7: The performance of two algorithms (sum received power versus the minimum altitude).

Particularly, the sum received power remains unchanged up to a certain distance between two ERs, but decreases thereafter. In fact, with increasing distance between ERs, the horizontal distance between UAV and ERs increases as well, and hence the sum received energy of ERs are decreasing due to the path loss effect. This phenomenon can also be observed with an increasing UAV altitude, vertical distance between ERs and $\mathrm{UAV}$, where the sum received power decreases due to the same reason. On the other hand, we have also investigated the sum received power versus the minimum UAV altitude, $h_{\text {min }}$, for $K=11$ energy receivers, for different UAV placement optimization schemes. In Fig. 7, it can be observed that the sum received power is the same up to a certain minimum altitude $h_{\min }$, i.e., $30<h_{\min }<50 \mathrm{~m}$, but drops afterwards. In fact, the minimum altitude below $50 \mathrm{~m}$ is not exceeding the optimal altitude of UAV $\frac{\sqrt{D_{\max }}}{\tan \Theta}$. Hence, the sum received power will not change with the minimum altitude below $50 \mathrm{~m}$ according to the equation (39). On the contrary, for the minimum altitude beyond $50 \mathrm{~m}$, the sum received power decreases since the optimal altitude should be with the range of the altitude requirements. Due to the security consideration, the optimal altitude of UAV in this case is thus set to $h_{\min }$.

Finally, we investigate the performance of hover-and-fly trajectory in UAV-enabled WPT system. We assume there exists a system with $K=11$ energy receivers that are randomly distributed within a 2D area of $500 \times 500 \mathrm{~m}^{2}$. For convenience, $\mathrm{B} \& \mathrm{~B}$ denotes branch and bound algorithm. The UAV will fly over two locations directly according to the designed flying plan. It can be seen in Fig. 8 that there are $\Gamma=4$ optimal locations, which are formed by ER 1-2, ER 3-5, ER 6-9, ER 10-11, respectively. The four hovering locations of UAV lie within the center of ERs that in the same serving area, respectively, in order to maximize the sum received power of ERs. Furthermore, we also investigate the harvested energy at $K=11$ ERs with different UAV placement optimization schemes. In Fig. 9, it can be seen that the received power values at different ERs are generally different. Particularly, the ERs that are closer to the UAV hovering locations can

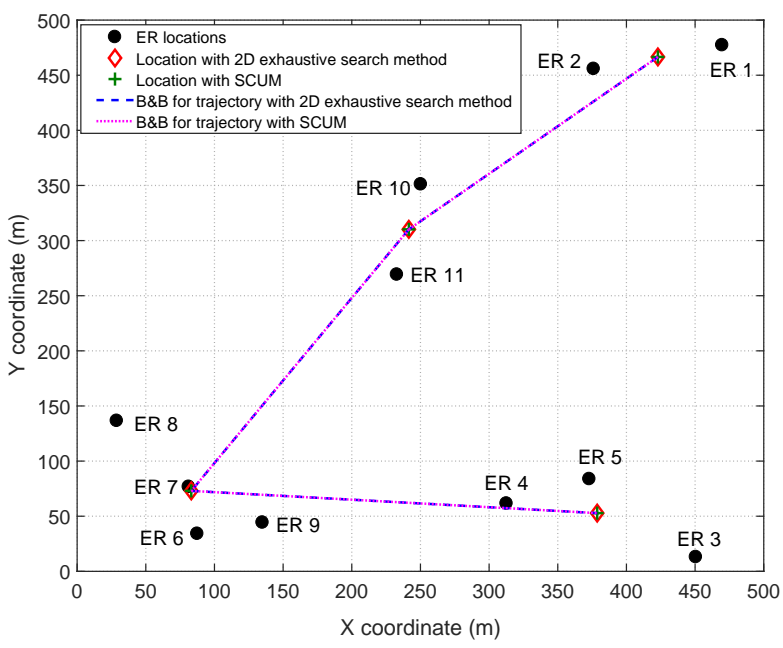

Fig. 8: Trajectory design for a UAV-enabled WPT system with $K=$ 11 ERs.

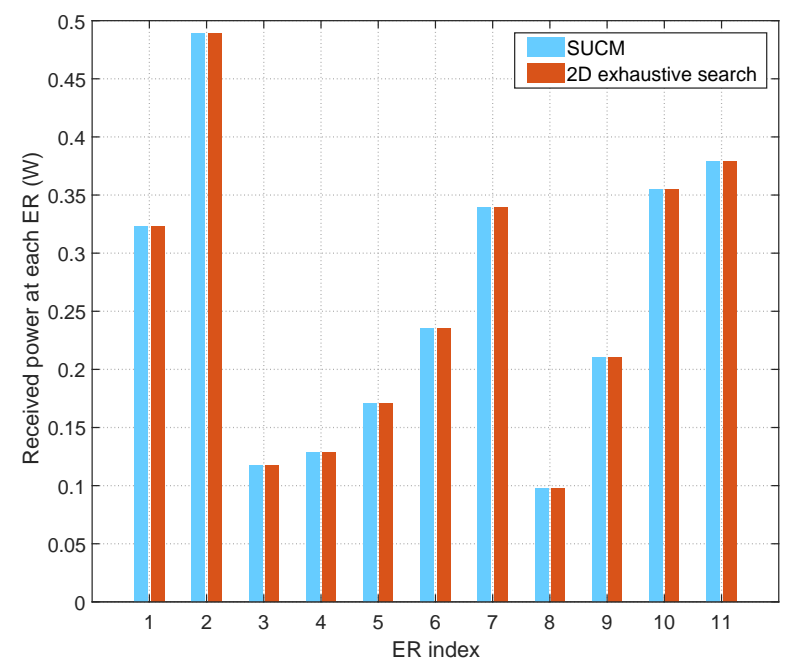

Fig. 9: The harvested energy at the $K=11$ ERs.

harvest more power than that of the edge ERs. For example, ER 7 receives more energy than ER 6, ER 8 and ER 9. This is because that ER 7 is near to the optimal hovering location than the others. However, this result further implies that the optimal hovering location solutions obtained by the low-complexity iterative algorithm can cause a near-far problem, and we will explore solutions to this problem in our future work. In fact, UAV's 3D position, beam gain and charging time will affect the energy harvested performance of ERs according to the equation (6).

\section{CONCLUSION}

In this paper, we study the energy harvesting optimization problem for UAV-enabled wireless power transfer system where a UAV acts as a wireless charger and delivers energy to a set of energy receivers. In particular, under the goal of maximizing energy harvesting of all ERs, a UAV equipped with antenna array is considered where multi-beams can be 
generated to serve multiple ERs simultaneously according to their position information. Due to the intractability and non-convex property of the energy harvesting optimization problem, we propose a low-complexity iterative algorithm to optimize UAV's 3D placement, beam pattern and charging time sequentially. Numerical results illustrate that the energy harvested performance of all ERs can be significantly improved by the proposed low-complexity iterative algorithm. In the future, joint UAV's 3D trajectory design and resource allocation scheme with considering multi-beam generation mechanism will be studied for UAV-enabled communication networks.

\section{APPENDIX A}

\section{Proof Of The Proposition 6}

Proof: We first rewrite the array factor of $M \times N$ antenna array in general as [20]

$$
\begin{aligned}
A F= & \sum_{\substack{n=1 \\
j(n-1)\left(2 \pi / \lambda d_{y} \sin (\theta) \sin (\phi)+\beta_{y}\right)}}^{N} I_{1 n} \times\left[\sum_{m=1}^{M} I_{m 1} e^{j(m-1)\left[2 \pi / \lambda d_{x} \sin (\theta) \cos (\phi)+\beta_{x}\right]}\right] .
\end{aligned}
$$

where the spacing and progressive phase shift between elements along the $x$-axis and $y$-axis are presented by $d_{x}, d_{y}$ and $\beta_{x}, \beta_{y}$, respectively. Assuming $\left(\theta_{*}, \phi_{*}\right)$ is the main-lobe direction of antenna array, we have

$$
\begin{aligned}
& \phi_{*}=\tan ^{-1}\left(\frac{\beta_{y} d_{x}}{\beta_{x} d_{y}}\right), \\
& \theta_{*}=\sin ^{-1}\left[\sqrt{\left(\frac{\beta_{x}}{\mathrm{k} d_{x}}\right)^{2}+\left(\frac{\beta_{y}}{\mathrm{k} d_{y}}\right)^{2}}\right],
\end{aligned}
$$

where $\mathrm{k}=2 \pi / \lambda$. Based on the main-lobe direction, we have the elevation plane half-power beamwidth

$$
\Theta_{h, e}=\sqrt{\frac{1}{\cos ^{2}\left(\theta_{*}\right)\left[\Theta_{x *}^{-2} \cos ^{2}\left(\phi_{*}\right)+\Theta_{y *}^{-2} \sin ^{2}\left(\phi_{*}\right)\right]}},
$$

where $\Theta_{x *}$ and $\Theta_{y *}$ denote the half-power beamwidth of a broadside linear array of $M$ and $N$ elements, respectively. On the other hand, the side-lobe level for antenna array can be given

$$
S L L=20 \lg \frac{\left|A F_{\text {msl }}\right|}{\left|A F_{\text {max }}\right|},
$$

where $A F_{m s l}$ denotes the array factor of maximum sidelobe level and $A F_{\max }$ is the array factor of main-lobe peak intensity.

We note that (5), (63)-(66) are functions of $\boldsymbol{\beta}=$ $\left[\beta_{1 n}, \cdots, \beta_{m n}, \cdots, \beta_{M N}\right]^{T}, \beta_{m n}=(m-1) \beta_{x}+(n-1) \beta_{y}$. Therefore, we construct the multiobjective optimization problem with decision variables $\boldsymbol{\beta}$ as

$$
\begin{aligned}
& \min F(\boldsymbol{\beta})=\left(f_{1}(\boldsymbol{\beta}), f_{2}(\boldsymbol{\beta}), f_{3}(\boldsymbol{\beta})\right)^{T} \\
& \text { s.t. } \boldsymbol{\beta} \in \mathbf{R}^{M \times N},
\end{aligned}
$$

where $f_{1}(\boldsymbol{\beta})=S L L(\boldsymbol{\beta}), f_{2}(\boldsymbol{\beta})=\frac{1}{|\mathbf{E}(\theta, \phi)|}, f_{3}(\boldsymbol{\beta})=\frac{1}{\left|\Theta_{h, e}\right|}$. Therefore, this completes the proof of Proposition 6 .

\section{APPENDIX B \\ Proof OF The Proposition 7}

During the optimization process of MOEA/D based algorithm, it first randomly generates $N_{\text {pop }}$ current solutions $\boldsymbol{\beta}_{1}, \cdots, \boldsymbol{\beta}_{N_{p o p}}$. Based on the parent points $\boldsymbol{\beta}_{1}, \cdots, \boldsymbol{\beta}_{N_{p o p}}$, it randomly chooses two points from the parent points and generates the new points by DE algorithm. Then, these new points and the parent points jointly construct the non-dominated solution set $E P_{0}$ by eliminating dominated points. However, $E P_{0}$ is far away from the globally Pareto optimal solution $\left\{\boldsymbol{\beta}^{*}\right\}$. Subsequently, it follows the same process in the next iteration and constructs a new non-dominated solution set $E P_{1}$, which is closer to the Pareto optimal solution $\left\{\boldsymbol{\beta}^{*}\right\}$ than $E P_{0}$. This process is repeated until the new non-dominated solution set $E P_{i}$ with $i \rightarrow \infty$ achieves the Pareto optimal solution, i.e., $\lim _{i \rightarrow \infty} E P_{i}=E P^{*}, E P^{*} \in\left\{\boldsymbol{\beta}^{*}\right\}$. Therefore, this completes the proof of Proposition 7.

\section{REFERENCES}

[1] G. A. Akpakwu, B. J. Silva, G. P. Hancke, and A. M. Abu-Mahfouz, "A survey on 5G networks for the Internet of Things: Communication technologies and challenges," IEEE Access, vol. 6, pp. 3619-3647, 2018.

[2] J. Wang, L. Zhao, J. Liu, and N. Kato, "Smart resource allocation for mobile edge computing: A deep reinforcement learning approach," IEEE Trans. Emerg. Topics Comput., to be published.

[3] L. Zhao, J. Wang, J. Liu, and N. Kato, "Routing for crowd management in smart cities: A deep reinforcement learning perspective," IEEE Commun. Mag., vol. 57, no. 4, pp. 88-93, Apr. 2019.

[4] Q. Luo, Y. Cao, J. Liu, and A. Bensilmane, "Localization and navigation in autonomous driving: Threats and countermeasures," IEEE Wireless Commun., to be published.

[5] J. Wang, J. Liu, and N. Kato, "Networking and communications in autonomous driving: A survey," IEEE Commun. Surveys Tuts., vol. 21, no. 2, pp. 1243-1274, Dec. 2018.

[6] S. Li, L. D. Xu, and S. Zhao, "5G Internet of Things: A survey," J. Ind. Inf. Integr., vol. 10, pp. $1-9,2018$.

[7] Y. Xun, J. Liu, N. Kato, Y. Fang, and Y. Zhang, "Automobile driver fingerprinting: A new machine learning based authentication scheme," IEEE Trans. Ind. Informat, vol. 16, no. 2, pp. 1417-1426, Feb. 2020.

[8] J. Liu, H. Guo, J. Xiong, N. Kato, J. Zhang, and Y. Zhang, "Smart and resilient EV charging in SDN-enhanced vehicular edge computing networks," IEEE J. Sel. Areas Commun., to be published.

[9] Y. Li, Q. Luo, J. Liu, H. Guo, and N. Kato, "TSP security in intelligent and connected vehicles: Challenges and solutions," IEEE Wireless Commun., vol. 26, no. 3, pp. 125-131, Jun. 2019.

[10] H. Guo, J. Zhang, and J. Liu, "FiWi-enhanced vehicular edge computing networks: Collaborative task offloading," IEEE Veh. Technol. Mag., vol. 14, no. 1, pp. 45-53, Mar. 2019.

[11] H. Zhang, W. Tian, and J. Liu, "Improving EDCA for efficient channel access in vehicular communications," IEEE Commun. Mag., vol. 56, no. 10, pp. 72-77, Oct. 2018.

[12] H. Zhang, Q. Zhang, J. Liu, and H. Guo, "Fault detection and repairing for intelligent connected vehicles based on dynamic bayesian network model," IEEE Internet Things J., vol. 5, no. 4, pp. 2431-2440, Aug. 2018.

[13] J. Tang, D. K. C. So, N. Zhao, A. Shojaeifard, and K.-K. Wong, "Energy efficiency optimization with SWIPT in MIMO broadcast channels for Internet of Things," IEEE Internet Things J., vol. 5, no. 4, pp. 26052619, Aug. 2018.

[14] X. Lu, P. Wang, D. Niyato, D. I. Kim, and Z. Han, "Wireless networks with RF energy harvesting: A contemporary survey," IEEE Commun. Surveys Tuts., vol. 17, no. 2, pp. 757-789, 2nd Quart., 2015.

[15] Y. Zeng and R. Zhang, "Energy-efficient UAV communication with trajectory optimization," IEEE Trans. Wireless Commun., vol. 16, no. 6 , pp. 3747-3760, Jun. 2017.

[16] J. Xu, Y. Zeng, and R. Zhang, "UAV-enabled wireless power transfer: Trajectory design and energy optimization," IEEE Trans. Wireless Commun., vol. 17, no. 8, pp. 5092-5106, Aug. 2018. 
[17] L. Xie, J. Xu, and R. Zhang, "Throughput maximization for UAVenabled wireless powered communication networks," IEEE Internet Things J., vol. 6, no. 2, pp. 1690-1703, Apr. 2019.

[18] M. Alzenad, A. El-Keyi, F. Lagum, and H. Yanikomeroglu, "3-D placement of an unmanned aerial vehicle base station (UAV-BS) for energyefficient maximal coverage," IEEE Wireless Commun. Lett., vol. 6, no. 4, pp. 434-437, Aug. 2017.

[19] Z. Yang, C. Pan, M. Shikh-Bahaei, W. Xu, M. Chen, M. Elkashlan, and A. Nallanathan, "Joint altitude, beamwidth, location, and bandwidth optimization for UAV-enabled communications," IEEE Commun. Lett., vol. 22 , no. 8, pp. 1716-1719, Aug. 2018.

[20] C. A. Balanis, Antenna Theory: Analysis and Design. New York, NY, USA: Wiley, 2016.

[21] S. Lee and R. Zhang, "Distributed wireless power transfer with energy feedback," IEEE Trans. Signal Process., vol. 65, no. 7, pp. 1685-1699, Apr. 2017

[22] J. Xu and R. Zhang, "A general design framework for MIMO wireless energy transfer with limited feedback," IEEE Trans. Signal Process., vol. 64, no. 10, pp. 2475-2488, May. 2016.

[23] N. Rupasinghe, Y. Yapıcı, I. Güvenç, and Y. Kakishima, "Nonorthogonal multiple access for mmWave drone networks with limited feedback," IEEE Trans. Commun., vol. 67, no. 1, pp. 762-777, Jan. 2019.

[24] H. Tuy and F. A. Al-Khayyal, "Global optimization of a nonconvex single facility location problem by sequential unconstrained convex minimization,' J. Global Optim., vol. 2, no. 1, pp. 61-71, Mar. 1992.

[25] M. Jiang, Y. Li, Q. Zhang, and J. Qin, "Joint position and time allocation optimization of UAV-enabled wireless powered communication networks," IEEE Trans. Commun., vol. 67, no. 5, pp. 3806-3816, May. 2019.

[26] Q. Zhang and H. Li, "MOEA/D: A multi-objective evolutionary algorithm based on decomposition," IEEE Trans. Evol. Comput., vol. 11, no. 6, pp. 712-731, Dec. 2007.

[27] S. Boyd and L. Vandenberghe, Convex Optimization. Cambridge, U.K.: Cambridge Univ. Press, 2004.

[28] Y. Zeng, X. Xu, and R. Zhang, "Trajectory design for completion time minimization in UAV-enabled multicasting," IEEE Trans. Wireless Commun., vol. 17, no. 4, pp. 2233-2246, Apr. 2018.

[29] L. Zhu, J. Zhang, Z. Xiao, X. Cao, D. O. Wu, and X. Xia, "3-D beamforming for flexible coverage in millimeter-wave UAV communications," IEEE Wireless Commun. Lett., vol. 8, no. 3, pp. 837-840, Jun. 2019.

[30] I. Ahmed, H. Khammari, A. Shahid, A. Musa, K. S. Kim, E. De Poorter, and I. Moerman, "A survey on hybrid beamforming techniques in 5G: Architecture and system model perspectives," IEEE Commun. Surveys Tuts., vol. 20, no. 4, pp. 3060-3097, 4th Quart., 2018.

[31] X. Ding, B.-Z. Wang, and G.-Q. He, "Research on a millimeter-wave phased array with wide-angle scanning performance," IEEE Trans. Antennas Propag., vol. 61, no. 10, pp. 5319-5324, Oct. 2013.

[32] A. Farajzadeh, O. Ercetin, and H. Yanikomeroglu, "UAV data collection over NOMA backscatter networks: UAV altitude and trajectory optimization," in 2019 IEEE International Conference on Communications (ICC), May 2019, pp. 1-7.

[33] I. Tsevendorj, "Piecewise-convex maximization problems," J. Global Optim., vol. 21, no. 1, pp. 1-14, Sep. 2001.

[34] J. Borwein and A. S. Lewis, Convex Analysis and Nonlinear Optimization: Theory and Examples. New York, NY, USA: Springer-Verlag, 2006.

[35] R. T. Rockafellar, Convex Analysis. Princeton, NJ: Princeton Univ. Press, 1970.

[36] M. Herceg, M. Kvasnica, C. N. Jones, and M. Morari, "Multi-Parametric Toolbox 3.0," in Proc. Eur. Control Conf. (ECC), Jul. 2013, pp. 502510.

[37] R. Storn and K. Price, "Differential evolution: A simple and efficient heuristic for global optimization over continuous spaces," J. Global Optimization, vol. 11, no. 4, pp. 341-359, Dec. 1997.

[38] C. E. Miller, A. Tucker, and R. Zemlin, "Integer Programming Formulation of Traveling Salesman Problems," J. ACM, vol. 7, pp. 326-329, Oct. 1960.

[39] K. N. Singh and D. L. van Oudheusden, "A branch and bound algorithm for the traveling purchaser problem," Eur. J. Oper. Res., vol. 97, no. 3, pp. 571-579, 1997. 